\title{
Polymer depletion profiles around nonspherical colloidal particles
}

E. Eisenriegler, and A. Bringer

Citation: The Journal of Chemical Physics 127, 034904 (2007); doi: 10.1063/1.2751193

View online: https://doi.org/10.1063/1.2751193

View Table of Contents: http://aip.scitation.org/toc/jcp/127/3

Published by the American Institute of Physics

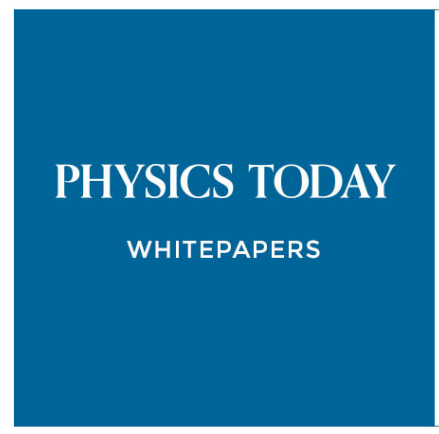




\title{
Polymer depletion profiles around nonspherical colloidal particles
}

\author{
E. Eisenriegler and A. Bringer \\ Institut für Festkörperforschung, Forschungszentrum Jülich, D-52425 Jülich, Germany
}

(Received 15 May 2007; accepted 29 May 2007; published online 19 July 2007)

\begin{abstract}
We study the effect of chain self-avoidance on the polymer density profiles that are induced by a single colloidal particle of nonspherical shape such as an ellipsoid, a dumbbell, or a lens in a solution of nonadsorbing polymers. For colloid sizes $\sigma$ much smaller than the size $\mathcal{R}_{x}$ of the polymers, we observe a pronounced difference between ideal and self-avoiding chains. In the case of ideal polymers, the surfaces of constant density always have the same character as the surface of the particle, e.g., are oblate for an oblate ellipsoid. In the self-avoiding case, however, the character changes with increasing distance $r$ from the particle, and an oblate particle induces prolate surfaces of constant density if $\sigma \ll r \ll \mathcal{R}_{x}$. For $\sigma \ll r, \mathcal{R}_{x}$, the isotropic and anisotropic contributions to the densities factor into a dependence on the particle size and shape and a dependence on $r, \mathcal{R}_{x}$. The latter is determined by distance distributions within a chain in the absence of the particle. For self-avoiding polymers in two spatial dimensions, exact density profiles are derived for $\sigma, r \ll \mathcal{R}_{x}$, which explicitly show the above-mentioned change of the contours of constant density. (C) 2007 American Institute of Physics. [DOI: 10.1063/1.2751193]
\end{abstract}

\section{INTRODUCTION}

Nonadsorbing polymers induce an attractive depletion interaction between colloidal particles, ${ }^{1}$ which is of considerable practical and scientific interest. ${ }^{2}$ In a previous paper ${ }^{3}$ we analyzed the effective interaction between a nonspherical particle and a wall, which depends on both the particle-wall distance and the orientation of the particle. Ideal ${ }^{4}$ and selfrepelling polymer chains were considered.

Here we study the simpler case of a single nonspherical particle, more amenable to a comparison with computer simulations. We find a remarkable difference between the anisotropic density depletion profiles that the particle induces in a dilute solution of ideal chains and self-repelling chains.

As in Ref. 3 we consider particles with an axis of rotational symmetry and with inversion symmetry about the center, such as ellipsoids, dumbbells, ${ }^{5}$ and lenses. An ellipsoid has diameters $D_{\|}$and $D_{\perp}$ parallel and perpendicular to the axis and is prolate if $D_{\|}>D_{\perp}$ and oblate if $D_{\|}<D_{\perp}$. A dumbbell is formed by two spheres of equal size that intersect at an angle $\alpha$ along a circle with diameter $\mathcal{D}$.

We concentrate on the case ${ }^{6}$ of "nanoparticles" with a size $\sigma$ that is mesoscopic but much smaller than the size $\mathcal{R}_{x}$ of the polymers. The latter is defined via the mean square end-to-end distance $\mathcal{R}^{2} \equiv d \mathcal{R}_{x}^{2}$ of the polymer chain, where $d$ is the spatial dimension. We analyze the bulk-normalized densities $\mathcal{M}(\mathbf{r})$ and $\mathcal{E}(\mathbf{r})$ of chain monomers and chain ends that are induced by the particle with center at the origin. In Sec. II we consider the small particle limit where $\sigma \ll r, \mathcal{R}_{x}$, and in Sec. III the long chain limit where $\sigma, r \ll \mathcal{R}_{x}$.

\section{SMALL PARTICLE LIMIT}

The interaction of a small particle with polymers can be described by a multipole-type expansion. ${ }^{3}$ The leading iso- tropic and anisotropic behaviors of the densities $\mathcal{E}$ and $\mathcal{M}$ induced by the particle in the dilute solution can be expressed in terms of particle amplitudes,

$$
I=\sigma^{x_{m}} \bar{I}, \quad N=\sigma^{d} \bar{N}, \quad N^{\prime}=\sigma^{x_{m}+2} \bar{N}^{\prime},
$$

where $\bar{I}, \bar{N}$, and $\bar{N}^{\prime}$ are dimensionless and depend on the shape of the particle, and distributions

$$
\eta(\varrho), \quad \varphi(\varrho), \quad \mu(\varrho) ; \quad \varrho=\hat{\mathbf{r}} / \mathcal{R}_{x}
$$

of the distance $\hat{\mathbf{r}}$ between the two ends, between an end and any monomer, and between any two monomers of a single chain in free space, without the particle. Here we consider the scaling limit, i.e., a large number $M$ of monomers per chain, and assume the normalizations

$$
\int \mathrm{d} \varrho \eta(\varrho)=\int \mathrm{d} \varrho \varphi(\varrho)=\int \mathrm{d} \varrho \mu(\varrho)=1 .
$$

For ellipsoids, dumbbells, and lenses, explicit expressions ${ }^{7}$ for the particle amplitudes [Eq. (2.1)] are given in Ref. 3 .

We introduce polymer critical exponents $x_{e}$ and $x_{m}$ that are related to the power law exponents ${ }^{4}$ in the molecular weight dependence $M^{\nu}$ of $\mathcal{R}_{x}$ and $M^{\gamma-1}$ of the partition function of a polymer with one end fixed in free space by

$$
2 x_{e}=d-\frac{\gamma}{\nu}, \quad x_{m}=d-\frac{1}{\nu} .
$$

The leading isotropic contribution to the densities $\mathcal{E}$ and $\mathcal{M}$ is given by

$$
\left\{\mathcal{E}_{\text {iso }}(\mathbf{r}), \mathcal{M}_{\text {iso }}(\mathbf{r})\right\}-1=-I \mathcal{R}_{x}^{-x_{m}}\left\{\varphi\left(\frac{r}{\mathcal{R}_{x}}\right), \mu\left(\frac{r}{\mathcal{R}_{x}}\right)\right\},
$$

and the leading anisotropic contribution by the sum of 


$$
\begin{aligned}
\left\{\mathcal{E}_{\text {aniso }}(\mathbf{r}), \mathcal{M}_{\text {aniso }}(\mathbf{r})\right\}= & -N \frac{r_{\|}^{2}}{r^{2}} \frac{1}{d-1}\left\{\frac{1}{\mathcal{R}_{x}^{d}}\left[\left(x_{e}-d\right) \eta\left(\frac{r}{\mathcal{R}_{x}}\right)-\left(2 x_{e}-d+r \frac{\mathrm{d}}{\mathrm{d} r}\right) \varphi\left(\frac{r}{\mathcal{R}_{x}}\right)\right]+\frac{d}{r^{d}} \int_{r / \mathcal{R}_{x}}^{\infty} \mathrm{d} \varrho \varrho^{d-1}\left[\left(x_{e}-d\right) \eta(\varrho)\right.\right. \\
& \left.-\left(2 x_{e}-d\right) \varphi(\varrho)\right], \frac{1}{\mathcal{R}_{x}^{d}}\left[2\left(x_{e}-d\right) \varphi\left(\frac{r}{\mathcal{R}_{x}}\right)-\left(2\left(x_{e}-d\right)+x_{m}+r \frac{\mathrm{d}}{\mathrm{d} r}\right) \mu\left(\frac{r}{\mathcal{R}_{x}}\right)\right] \\
& \left.+\frac{d}{r^{d}} \int_{r / \mathcal{R}_{x}}^{\infty} \mathrm{d} \varrho \varrho^{d-1}\left[2\left(x_{e}-d\right) \varphi(\varrho)-\left(2\left(x_{e}-d\right)+x_{m}\right) \mu(\varrho)\right]\right\},
\end{aligned}
$$

and

$$
\left\{\mathcal{E}_{\text {aniso }}^{\prime}(\mathbf{r}), \mathcal{M}_{\text {aniso }}^{\prime}(\mathbf{r})\right\}=-N^{\prime} \mathcal{R}_{x}^{-x_{m}} \frac{\partial^{2}}{\partial r_{\|}^{2}}\left\{\varphi\left(\frac{r}{\mathcal{R}_{x}}\right), \mu\left(\frac{r}{\mathcal{R}_{x}}\right)\right\},
$$

as we show in Appendix A. In the long chain limit $\mathcal{R}_{x} \rightarrow \infty$ the above expressions reduce to

$$
\begin{aligned}
& \left\{\mathcal{E}_{\text {iso }}(\mathbf{r}), \mathcal{M}_{\text {iso }}(\mathbf{r})\right\}-1 \rightarrow-I \frac{1}{r^{x_{m}}}\left\{c_{e}, c_{m}\right\}, \\
& \left\{\mathcal{E}_{\text {aniso }}(\mathbf{r}), \mathcal{M}_{\text {aniso }}(\mathbf{r})\right\} \rightarrow N \frac{r_{\|}^{2}}{r^{2}} \frac{1}{r^{d}} \frac{d}{d-1} \frac{\Gamma(d / 2)}{2 \pi^{d / 2}}\left\{x_{e}, x_{m}\right\}, \\
& \left\{\mathcal{E}_{\text {aniso }}^{\prime}(\mathbf{r}), \mathcal{M}_{\text {aniso }}^{\prime}(\mathbf{r})\right\} \rightarrow-N^{\prime} \frac{r_{\|}^{2}}{r^{2}} \frac{1}{r^{x_{m}+2}} x_{m}\left(x_{m}+2\right)\left\{c_{e}, c_{m}\right\} .
\end{aligned}
$$

Here $r_{\|}$is the component of $\mathbf{r}$ parallel to the particle axis, and $c_{e}$ and $c_{m}$ are the positive amplitudes in the short distance asymptotic forms $\varphi(\varrho) \rightarrow c_{e} \varrho^{-x_{m}}$ and $\mu(\varrho) \rightarrow c_{m} \varrho^{-x_{m}}$ for $\varrho$ $\ll 1$. The leading long chain behavior [Eq. (2.9)] arises from the terms with integrals in Eq. (2.6) via normalizations (2.3). An isotropic term has been discarded on deriving Eq. (2.10) from Eq. (2.7). The exponents $x_{e}$ and $x_{m}$, the amplitudes $c_{e}$ and $c_{m}$, and the functions $\bar{I}, \bar{N}$, and $\bar{N}^{\prime}$ and $\eta, \varphi$, and $\mu$ are "universal," i.e., independent of the chemical microstructure of the chains but different for ideal and self-repelling chains.

The two coefficients $N$ and $N^{\prime}$ describing the anisotropic behavior are both positive for prolate particle shapes such as dumbbells and prolate ellipsoids and both negative for oblate shapes. This applies to ideal ${ }^{8}$ as well as self-repelling ${ }^{3}$ polymer chains. Visualizing the anisotropy of the density profiles by means of contour surfaces of constant density, we find that the contribution [Eq. (2.10)] from $N^{\prime}$ leads to an ellipsoidal contour with the same prolate/oblate character as the particle surface, while the contribution [Eq. (2.9)] from $N$ has the opposite character. ${ }^{9}$

For self-repelling chains, $\nu>1 / 2$, and thus $(\sigma / r)^{d}$ $\gg(\sigma / r)^{x_{m}+2}$ for small $\sigma / r$, so that the $N$ term in Eq. (2.9) dominates, and the $N^{\prime}$ term in Eq. (2.10) can, in general, be neglected. This leads to the remarkable result that for $\sigma \ll r$ $\ll \mathcal{R}_{x}$ the contour surfaces of an oblate particle are prolate and vice versa if the chains are self-repelling.

However, for ideal chains, where $x_{m}=d-2$, both aniso- tropic contributions [Eqs. (2.9) and (2.10)] are of the same order, and their sum has the sign of the $N^{\prime}$ term in Eq. (2.10), so that the contour surfaces have the same character as the particle shape. This is discussed in more detail at the end of Appendix A, where we also present explicit expressions for the distance distributions $\eta, \varphi$, and $\mu$ and the amplitudes $c_{e}$ and $c_{m}$ of ideal chains.

\section{LONG CHAIN LIMIT}

On approaching the particle surface, $\mathcal{E}$ and $\mathcal{M}$ vanish in the scaling limit, i.e., the particle surface is a contour surface with density zero. In the case of self-repelling chains it is interesting to explicitly see the changeover to the contour surfaces of opposite prolate/oblate character, mentioned above, as the distance from the particle increases. To this end consider the long chain limits, ${ }^{10}$

$$
\begin{aligned}
\hat{\mathcal{E}}(\mathbf{r}) & \equiv \lim _{\mathcal{R}_{x} \rightarrow \infty} \mathcal{E}(\mathbf{r}) \\
& =\lim _{\widetilde{\mathbf{r}} \rightarrow \infty}\langle\Phi(\mathbf{r}) \Phi(\widetilde{\mathbf{r}})\rangle_{\text {part }} /\langle\Phi(0) \Phi(\widetilde{\mathbf{r}})\rangle_{\text {bulk }}
\end{aligned}
$$

and

$$
\begin{aligned}
\hat{\mathcal{M}}(\mathbf{r}) & \equiv \lim _{\mathcal{R}_{x} \rightarrow \infty} \mathcal{M}(\mathbf{r}) \\
& =\lim _{\widetilde{\mathbf{r}} \rightarrow \infty}\langle\Psi(\mathbf{r}) \Psi(\widetilde{\mathbf{r}})\rangle_{\text {part }} /\langle\Psi(0) \Psi(\widetilde{\mathbf{r}})\rangle_{\text {bulk }},
\end{aligned}
$$

of $\mathcal{E}$ and $\mathcal{M}$ for $\mathcal{R}_{x} \rightarrow \infty$, which using the polymer-magnet equivalence, ${ }^{4}$ can be obtained from correlation functions \langle\rangle$_{\text {part }}$ and \langle\rangle$_{\text {bulk }}$ at the critical point of the zero component vector field theory, in the presence and absence of the particle. Here $\Phi$ is the order-parameter density, and $\Psi$ is proportional $^{3}$ to the energy density in the field theory.

For $r \gg \sigma$ the three leading contributions to the right hand sides of Eqs. (3.1) and (3.2) are given by the right hand sides of Eqs. (2.8)-(2.10), as expected and shown in Appendix B.

Results for arbitrary $\mathbf{r}$ are obtained for the monomer density $\hat{\mathcal{M}}$ of self-repelling chains in $d=2$ spatial dimensions outside an ellipse, a dumbbell of two intersecting circles, and a lens with two circular surface lines, using the corresponding correlation functions $\langle\Psi \Psi\rangle_{\text {part }}$ given ${ }^{11}$ in Appendix A of Ref. 3. 


\section{A. Ellipsoids}

For the ellipse in $d=2$ with diameters $D_{\|}<D_{\perp}$,

$$
\hat{\mathcal{M}}_{\mathrm{ell}}=\left[\frac{\tilde{E}^{2}}{4\left(E^{2}+H^{2}\right)}\right]^{1 / 3} q_{\mathrm{ell}}^{2 / 3} \mathcal{G}\left(q_{\mathrm{ell}}\right) \text {. }
$$

Here,

$$
\widetilde{E}=E+\sqrt{1+E^{2}},
$$

$E$ and $H$ are elliptic coordinates given by

$$
\begin{aligned}
& 2 f \sqrt{1+E^{2}}=\sqrt{r_{\|}^{2}+\left(\left|r_{\perp}\right|+f\right)^{2}}+\sqrt{r_{\|}^{2}+\left(\left|r_{\perp}\right|-f\right)^{2}}, \\
& 2 f \sqrt{1-H^{2}}=\sqrt{r_{\|}^{2}+\left(\left|r_{\perp}\right|+f\right)^{2}}-\sqrt{r_{\|}^{2}+\left(\left|r_{\perp}\right|-f\right)^{2}},
\end{aligned}
$$

with the interfocal distance,

$$
2 f=\sqrt{D_{\perp}^{2}-D_{\|}^{2}},
$$

and

$$
q_{\mathrm{ell}}=\frac{1}{g \widetilde{E}^{2}-1}, \quad g=\frac{D_{\perp}-D_{\|}}{D_{\perp}+D_{\|}} .
$$

The quantity $q$ tends to $+\infty$ and 0 if the distance of $\mathbf{r}$ from the particle surface tends, respectively, to 0 and $\infty$. For moderate distances,

$$
\mathcal{G}(q)=\frac{8 \pi}{45 \sqrt{3}} \frac{1}{\xi}{ }_{3} F_{2}\left(\frac{1}{3}, \frac{2}{3}, \frac{7}{3} ; \frac{11}{6}, 2 ;-\frac{1}{4 \xi}\right), \quad \xi=q(1+q),
$$

where ${ }_{3} F_{2}$ is a generalized hypergeometric function. ${ }^{12}$ For larger distances, $\mathcal{G}$ follows from analytic continuation. In particular, for $q \rightarrow 0$,

$$
\mathcal{G}(q) \rightarrow \xi^{-2 / 3}\left[1-\xi^{1 / 3}\left|B_{2}\right|+2 \xi / 3+\mathcal{O}\left(\xi^{4 / 3}\right)\right],
$$

where $\left|B_{2}\right|=1.05$ (see footnote 24 in Ref. 13). The leading anisotropic behavior,

$$
\hat{\mathcal{M}}_{\text {ell,aniso }}=-\frac{1}{3} f^{2} \frac{r_{\|}^{2}}{r^{4}}
$$

of $\hat{\mathcal{M}}_{\text {ell }}$ at large distances comes from the first factor on the right hand side of Eq. (3.3), which tends to $1-H^{2} /\left(3 E^{2}\right)$, while $q^{2 / 3} \mathcal{G}$ tends to 1 . Equation (3.10) is consistent with Eq. (2.9), since the particle amplitude $N$ is negative and given by $N=-(\pi / 2) g\left[\left(D_{\perp}+D_{\|}\right) / 2\right]^{2}$ [see Eq. (3.6) in Ref. 3]. Unlike the particle surface, the contour surface $\hat{\mathcal{M}}_{\text {ell }}=$ const, with const slightly below 1 , extends farther in the $\|$ than in the $\perp$ direction. For an ellipse with $D_{\|} / D_{\perp}=1 / 3$ and a "needle" with $D_{\|}=0$ in $d=2$, this is shown in Fig. 1. As a consequence, the two graphs $\hat{\mathcal{M}}\left(r_{\perp}=r, r_{\|}=0\right)$ and $\hat{\mathcal{M}}\left(r_{\perp}=0, r_{\|}=r\right)$ must cross at a certain $r=r_{c}$. For the needle this is shown in Fig. 2.

The density profile [Eq. (3.3)] for self-repelling polymers, which depends on both coordinates $E$ and $H$, should be compared with the corresponding profile for ideal chains around ellipsoidal particles. In this case the surfaces of constant monomer density are ellipsoids confocal with the particle surface, since the profile only depends on $E$. For example, for an oblate ellipsoid ${ }^{1,8}$ in $d=3$,

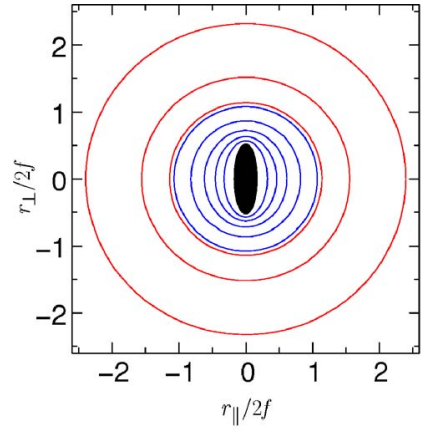

(a)

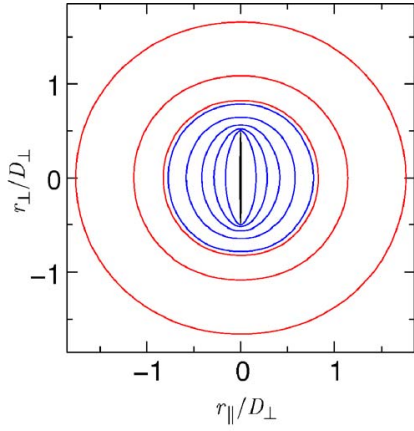

(b)
FIG. 1. (a) Density contour lines for $\hat{\mathcal{M}}=0,0.1,0.2,0.3,0.4,0.49,0.51$, 0.6 , and 0.7 of long self-avoiding chains in $d=2$ outside a nonadsorbing elliptical particle with aspect ratio $D_{\|} / D_{\perp}=1 / 3$. Note that the contour $\hat{\mathcal{M}}$ $=0.7$ extends farther in the $\|$ than in the $\perp$ direction, i.e., has an anisotropy opposite to that of the particle surface where $\hat{\mathcal{M}}=0$. (b) Same for a needle of length $D_{\perp}$ and with $D_{\|}=0$ in $d=2$.

$$
\hat{\mathcal{M}}_{\text {ell }}^{\text {(ideal) }}=\left[1-\frac{\arctan (1 / E)}{\arctan \sqrt{\left(D_{\perp} / D_{\|}\right)^{2}-1}}\right]^{2} .
$$

Here $\left|r_{\perp}\right|$ in Eq. (3.5) is the modulus of the two-dimensional component of $\mathbf{r}$ perpendicular to the rotation axis. The contour surfaces $\hat{\mathcal{M}}_{\text {ell }}^{\text {(ideal) }}=$ const for an ellipsoid with $D_{\|} / D_{\perp}$ $=1 / 3$ and a circular disk with $D_{\|}=0$ in $d=3$ are shown in Fig. 3.

\section{B. Dumbbells and lenses}

For the dumbbell with $0 \leqslant \alpha \leqslant \pi$ and the lens with $\pi$ $\leqslant \alpha \leqslant 2 \pi$ shown in Figs. 1 and 2 of Ref. 3,

$$
\begin{aligned}
\hat{\mathcal{M}}_{\mathrm{db} / 1}(\mathbf{r})= & \left(\frac{\pi}{\alpha}\right)^{4 / 3}\left\{\left|W^{2}-1\right| \operatorname{Re}\left[\left(\frac{\theta_{+} \bar{\theta}_{-}}{\bar{\theta}_{+} \theta_{-}}\right)^{\pi /(2 \alpha)}\right]\right\}^{-2 / 3} \\
& \times \mathcal{G}\left(q_{\mathrm{db} / 1}\right),
\end{aligned}
$$

where

$$
W=\frac{r_{\perp}+i r_{\|}}{\mathcal{D} / 2}, \quad \bar{W}=\frac{r_{\perp}-i r_{\|}}{\mathcal{D} / 2},
$$

and where $\mathcal{D}$ is the distance between the intersection points of the two circular surface lines,

$$
\begin{aligned}
& \theta_{ \pm}=1 \pm \frac{1}{W}, \quad \bar{\theta}_{ \pm}=1 \pm \frac{1}{\bar{W}}, \\
& q_{\mathrm{db} / 1}=\frac{\left|\left(\theta_{+}\right)^{\pi / \alpha}-\left(\theta_{-}\right)^{\pi / \alpha}\right|^{2}}{4 \operatorname{Re}\left[\left(\theta_{+} \bar{\theta}_{-}\right)^{\pi / \alpha}\right]},
\end{aligned}
$$

and $\mathcal{G}$ is the function in Eq. (3.8). The intersection points are located at $r_{\perp}= \pm \mathcal{D} / 2$ and $r_{\|}=0$, and $R=\mathcal{D} /(2 \sin (\alpha / 2))$ is the radius of the circular surface lines.

For the special case $\alpha=0$ of a dumbbell of two touching circles of radius $R$, Eqs. (3.12)-(3.14) lead to the result given in Eqs. (3.4) and (3.5) of Ref. 13 and to the density contour lines given in Fig. 4 of the present paper. The lower curve in the numerical plot in Fig. 1 of Ref. 13 is erroneous and is corrected in Fig. 5 of the present paper. The crossing of 


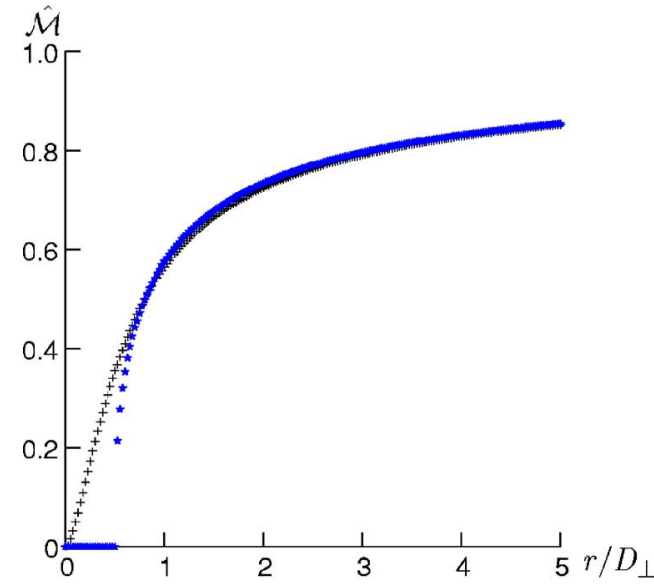

(a)

$\hat{\mathcal{M}}\left(r_{\perp}=\rho R, 0\right)$ and $\hat{\mathcal{M}}\left(0, r_{\|}=\rho R\right)$, which appears in Fig. 5 slightly below $\rho=5$, implies contours $\hat{\mathcal{M}}=$ const that are more extended along the $r_{\|}$axis and the $r_{\perp}$ axis for const $\ll 1$ and const $\rightarrow 1$, respectively. This agrees with Fig. 4 and is consistent with Eq. (2.9), since the particle amplitude $N$ $=-\mathcal{D}^{2}(\pi / 6)\left[1-(\pi / \alpha)^{2}\right]$ for the dumbbell or lens ${ }^{3}$ is positive, $N=R^{2} \pi^{3} / 6$, for $\alpha=0$.

The special cases of a needle of length $l$ and a circle of radius $R$ in $d=2$ correspond to $D_{\perp}=l, D_{\|}=0$ and $D_{\perp}=D_{\|}$ $=2 R$ in Eq. (3.3) and to $\mathcal{D}=l, \alpha=2 \pi$ and $\mathcal{D}=2 R, \alpha=\pi$ in Eq. (3.12). The result for the circle is given in Eq. (1) of the second paper in Ref. 10.

For small distances from the particle surface, $\mathcal{M}_{\text {ell }}$ and $\mathcal{M}_{\mathrm{dbl}}$ are proportional to the distance raised to the power $1 / \nu$, with $1 / \nu=4 / 3$ in $d=2$.

\section{SUMMARY AND CONCLUDING REMARKS}

We have shown that the chain self-avoidance has a pronounced effect on the anisotropic density profiles that are induced by a nonspherical colloidal particle in a polymer solution. In particular, we considered the densities $\mathcal{M}$ of chain monomers and $\mathcal{E}$ of chain ends induced by mesoscopic particles with the shapes of ellipsoids, dumbbells, and lenses and with sizes $\sigma$ much smaller than the polymer size $\mathcal{R}_{x}$.

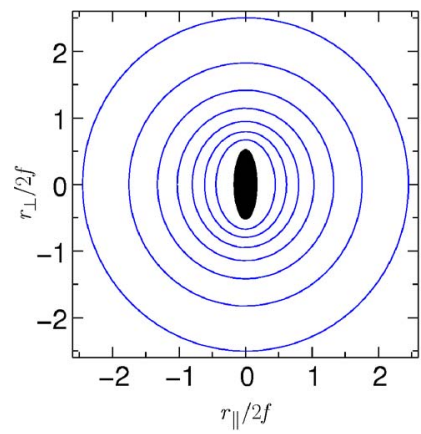

(a)

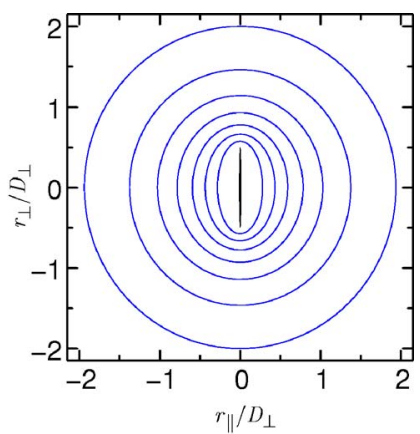

(b)
FIG. 3. (a) Density contour surfaces for $\hat{\mathcal{M}}=0,0.1,0.2,0.3,0.4,0.5,0.6$, and 0.7 of long ideal chains in $d=3$ outside a nonadsorbing oblate ellipsoidal particle with aspect ratio $D_{\|} / D_{\perp}=1 / 3$. Shown are contour lines in a plane $\left(r_{\perp}, r_{\|}\right)$that contains the particle axis at $r_{\perp}=0$. (b) Same for a circular plate in $d=3$ with $D_{\|}=0$. All the contours extend farther in the $\perp$ than in the II direction and have the same type of anisotropy as the particle surface.
For $\sigma \ll r, \mathcal{R}_{x}$ the profiles in dilute solution are determined by three universal amplitudes which characterize the size and shape of the particle and three distance distributions for a single chain without the particle present. This is shown in Eqs. (2.5)-(2.7) of Sec. II. For self-avoiding polymers in two spatial dimensions, we analyzed $\mathcal{M}$ in the regime $\sigma, r$ $\ll \mathcal{R}_{x}$, which encompasses both $\mathbf{r}$ close to the particle surface and $r$ much larger than the particle size.

The densities are depleted near the particle, where the polymer fluctuations are impeded. The anisotropic shape of the obstacle has two opposing effects on the densities. Consider, e.g., an oblate particle with the shape of a pancake or circular disk of diameter $l$ and compare a point $\mathbf{r}_{p}=\left(\mathbf{r}_{\perp}, 0\right)$, where $r_{\perp}=r>l / 2$, in the plane of the disk with a point $\mathbf{r}_{a}$ $=(0, r)$ on its axis. Both points have the same distance $r$ from the particle center. (i) The closest distance to the obstacle in the case of $\mathbf{r}_{a}$ is $r$ and is larger than the closest distance $r$ $-l / 2$ in the case of $\mathbf{r}_{p}$. Therefore, one might expect that the densities at $\mathbf{r}_{a}$ are larger than at $\mathbf{r}_{p}$. (ii) However, the obstacle appears wider from $\mathbf{r}_{a}$ than from $\mathbf{r}_{p}$ suggesting a trend in the opposite direction. Actually, we find for $\sigma \ll r \ll \mathcal{R}_{x}$ that the

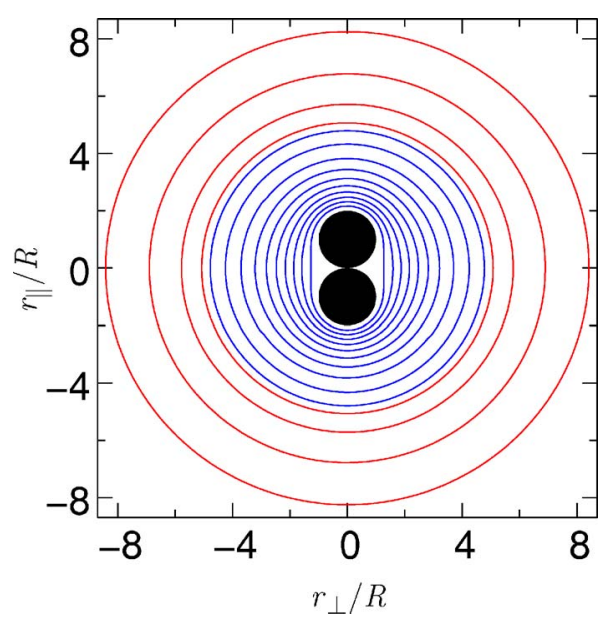

FIG. 4. Density contour lines for $\hat{\mathcal{M}}=0,0.05,0.1,0.15,0.2,0.25,0.3,0.35$, $0.4,0.45,0.49,0.51,0.55,0.6$, and 0.65 about a dumbbell particle of two touching circles of radius $R$ in $d=2$. The contour $\hat{\mathcal{M}}=0.65$ extends farther in the $\perp$ than $\|$ direction, i.e., has an anisotropy opposite to the particle surface where $\hat{\mathcal{M}}=0$. 


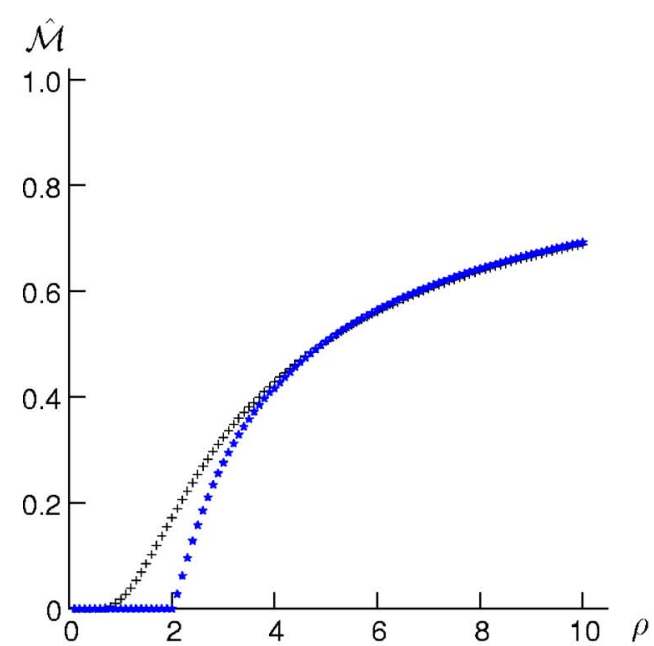

(a)

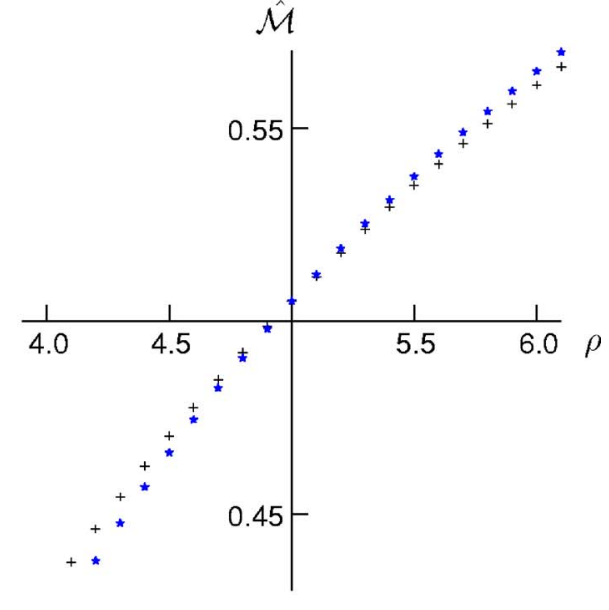

(b) trends (i) and (ii) prevail in the absence and presence, respectively, of chain self-avoidance. This applies to general oblate particle shapes and also to prolate shapes, where the roles of $\mathbf{r}_{p}$ and $\mathbf{r}_{a}$ are interchanged [see Eqs. (2.9) and (2.10), the last two paragraphs in Sec. II, and the comparison of selfavoiding and ideal chain results in Eqs. (3.3) and (3.11)].

Figures 1, 3, and 4 illustrate this effect in terms of contour surfaces of constant density. The anisotropy of contour surfaces with $\sigma \ll r \ll \mathcal{R}_{x}$ is opposite to that of the particle surface if the chains are self-avoiding. The $r$ dependencies of the densities of self-avoiding chains along $\mathbf{r}_{p}$ and $\mathbf{r}_{a}$ cross at a certain $r=r_{c}$, as shown in Figs. 2 and 5. The value of the bulk-normalized monomer density $\mathcal{M}$ at the crossing is very close to $1 / 2$ for all our particle shapes in two dimensions.

It would be interesting to check our predictions for polymer density profiles about anisotropic particles in simulations. ${ }^{6}$ In the case of the needle in $d=2$ considered in Fig. 1(b), one could consider self-avoiding walks on a quadratic lattice with the lattice sites along a finite straight line excluded.

\section{ACKNOWLEDGMENT}

It is a pleasure to thank T. W. Burkhardt for useful discussions.

\section{APPENDIX A: SMALL PARTICLE EXPANSION FOR DENSITY PROFILES}

Using the polymer-magnet equivalence, ${ }^{4}$ the polymer densities around the particle can be expressed as

$$
\begin{aligned}
& \mathcal{E}(\mathbf{r})=\mathcal{L}\langle\Phi(\mathbf{r}) \phi\rangle_{\text {part }} / \Xi_{\text {bulk }}, \\
& \mathcal{M}(\mathbf{r})=\mathcal{L}\left\langle\Psi(\mathbf{r}) \phi^{2}\right\rangle_{\text {part }} / \mathcal{L}\left\langle\Psi(\mathbf{r}) \phi^{2}\right\rangle_{\text {bulk }},
\end{aligned}
$$

and the distance distributions of a free chain as

$$
\begin{aligned}
& \eta\left(r / \mathcal{R}_{x}\right)=\mathcal{R}_{x}^{d} \mathcal{L}\langle\Phi(\mathbf{r}) \Phi(0)\rangle_{\text {bulk }} / \Xi_{\text {bulk }}, \\
& \varphi\left(r / \mathcal{R}_{x}\right)=\mathcal{R}_{x}^{d-1 / \nu} \mathcal{L}\langle\Psi(\mathbf{r}) \Phi(0) \phi\rangle_{\text {bulk }} / \Xi_{\text {bulk }}, \\
& \mu\left(r / \mathcal{R}_{x}\right)=\mathcal{R}_{x}^{d-2 / \nu} \mathcal{L}\left\langle\Psi(\mathbf{r}) \Psi(0) \phi^{2}\right\rangle_{\text {bulk }} / \Xi_{\text {bulk }},
\end{aligned}
$$

in terms of correlation functions of the $n$-vector model in the limit $n \rightarrow 0$. The Laplace transform $\mathcal{L}$ relates the temperature deviation from the critical point of the $n$-vector model to the chain length and is defined in Eq. (2.5) of Ref. 3, the energydensity like quantity $\Psi$ is defined in Eqs. (2.4) and (2.8) of Ref. 3, and

$$
\phi=\int \mathrm{d} \mathbf{r}^{\prime} \Phi\left(\mathbf{r}^{\prime}\right), \quad \Xi_{\text {bulk }}=\mathcal{L}\langle\Phi(\mathbf{r}) \phi\rangle_{\text {bulk }} .
$$

Normalization (2.3) of $\varphi$ and $\mu$ follows from Eq. (C2) in Ref. 3.

Eqations (A2) imply the relations,

$$
c_{e}=C_{\Phi \Phi \Psi} / B_{\Phi}, \quad c_{m}=C_{\Psi \Psi \Psi} / B_{\Psi},
$$

between the polymer short distance amplitudes $c_{e}$ and $c_{m}$ defined below Eq. (2.10) and the amplitudes ${ }^{14}$ in the operator product expansions $\Psi(\mathbf{r}) \Phi(0) \rightarrow\left(C_{\Phi \Phi \Psi} / B_{\Phi}\right) r^{-x_{m}} \Phi(0)$ and $\Psi(\mathbf{r}) \Psi(0) \rightarrow\left(C_{\Psi \Psi \Psi} / B_{\Psi}\right) r^{-x_{m}} \Psi(0)$ of the $n$-vector field theory.

The operator expansion, ${ }^{3,7}$

$$
\begin{aligned}
\exp \left(-\mathcal{H}_{\text {part }}\right) \propto & 1-I \Psi\left(\mathbf{r}_{P}\right)-N T_{\|\|}\left(\mathbf{r}_{P}\right) \\
& -N^{\prime} \partial_{\|}^{2} \Psi\left(\mathbf{r}_{P}\right)+\cdots
\end{aligned}
$$

for the Boltzmann factor of a small particle embedded at $\mathbf{r}_{P}$ in the $n \rightarrow 0$ model allows us to relate the leading isotropic and anisotropic contributions in $\mathcal{E}$ and $\mathcal{M}$ to $\eta, \varphi$, and $\mu$, as given in Eqs. (2.5)-(2.7). Here $\partial_{\|}$and $T_{\|\|}$denote the directional derivative and the diagonal component of the stress tensor along the direction of the particle axis.

Consider first the leading isotropic contributions. Replacing $\exp \left(-\mathcal{H}_{\text {part }}\right)$ in the statistical weight of Eq. (A1) by the first two terms on the right hand side of expansion (A5) and using Eqs. (A2) and (2.4) leads to relation (2.5). In the case of $\mathcal{M}_{\text {iso }}$ one also uses that $\mathcal{L}\left\langle\Psi \phi^{2}\right\rangle_{\text {bulk }}$ equals $\mathcal{R}_{x}^{1 / \nu} \Xi_{\text {bulk }}$, as follows from translational invariance of bulk averages and Eq. (C2) in Ref. 3. Relation (2.7) follows similarly.

To derive the relation for $\mathcal{E}_{\text {aniso }}$ in Eq. (2.6), we consider 


$$
\mathcal{R}_{x}^{d} \frac{\mathcal{L}\left\langle T_{k l}\left(\mathbf{r}_{P}\right) \Phi(\mathbf{r}) \phi\right\rangle_{\text {bulk }}}{\Xi_{\text {bulk }}}=\frac{s_{k} s_{l}}{s^{2}} \tau_{e}(\varrho)+\delta_{k l} \tilde{\tau}_{e}(\varrho),
$$

where $\mathbf{s}=\mathbf{r}_{P}-\mathbf{r}$ and $\varrho=s / \mathcal{R}_{x}$. The right hand side of Eq. (A6) follows from translational and rotational symmetries of bulk averages. The universal functions $\tau_{e}$ and $\widetilde{\tau}_{e}$ are determined by the trace and continuity equations of the stress tensor $T_{k l}$ given in Eqs. (C8) and (C9) of Ref. 3 which yield

$$
\tau_{e}+d \widetilde{\tau}_{e}=-x_{e} \eta+\left(2 x_{e}+\varrho \frac{\mathrm{d}}{\mathrm{d} \varrho}\right) \varphi
$$

and

$$
(d-1) \tau_{e}+\varrho \frac{\mathrm{d}}{\mathrm{d} \varrho}\left(\tau_{e}+\tilde{\tau}_{e}\right)=-\varrho \frac{\mathrm{d}}{\mathrm{d} \varrho} \eta,
$$

respectively. To derive Eq. (A7), we have used Eq. (C3) in Ref. 3 and

$$
\frac{\mathrm{d} \ln \left[\Xi_{\text {bulk }}\left(\mathcal{R}_{x}\right)^{-x_{m}}\right]}{\mathrm{d} \ln \mathcal{R}_{x}}=-2 x_{e} .
$$

The sum of Eqs. (A7) and (A8) is a linear differential equation for $\tau_{e}+\tilde{\tau}_{e}$, with solution

$$
\begin{aligned}
\tau_{e}(\varrho)+\tilde{\tau}_{e}(\varrho)= & \varrho^{-d} \int_{\varrho}^{\infty} \mathrm{d} \bar{\varrho} \bar{\varrho}^{d-1}\left[\left(x_{e}+\bar{\varrho} \frac{\mathrm{d}}{\mathrm{d} \bar{\varrho}}\right) \eta(\bar{\varrho})\right. \\
& \left.-\left(2 x_{e}+\bar{\varrho} \frac{\mathrm{d}}{\mathrm{d} \bar{\varrho}}\right) \varphi(\bar{\varrho})\right] .
\end{aligned}
$$

Equations (A10) and (A8) lead to an explicit expression for the anisotropy coefficient $\tau_{e}$, which via Eq. (A6) implies the result for $\mathcal{E}_{\text {aniso }}$ in Eq. (2.6).

The result for $\mathcal{M}_{\text {aniso }}$ follows in a similar fashion by considering

$$
\mathcal{R}_{x}^{d} \frac{\mathcal{L}\left\langle T_{k l}\left(\mathbf{r}_{P}\right) \Psi(\mathbf{r}) \phi^{2}\right\rangle_{\text {bulk }}}{\mathcal{L}\left\langle\Psi(\mathbf{r}) \phi^{2}\right\rangle_{\text {bulk }}}=\frac{s_{k} s_{l}}{s^{2}} \tau_{m}(\varrho)+\delta_{k l} \widetilde{\tau}_{m}(\varrho) .
$$

Here,

$$
\begin{aligned}
& \tau_{m}+d \widetilde{\tau}_{m}=-2 x_{e} \varphi+\left(2 x_{e}-\frac{1}{\nu}+\varrho \frac{\mathrm{d}}{\mathrm{d} \varrho}\right) \mu, \\
& (d-1) \tau_{m}+\varrho \frac{\mathrm{d}}{\mathrm{d} \varrho}\left(\tau_{m}+\widetilde{\tau}_{m}\right)=-2 \varrho \frac{\mathrm{d}}{\mathrm{d} \varrho} \varphi,
\end{aligned}
$$

and

$$
\begin{aligned}
\tau_{m}(\varrho)+\widetilde{\tau}_{m}(\varrho)= & \varrho^{-d} \int_{\varrho}^{\infty} \mathrm{d} \bar{\varrho} \bar{\varrho}^{d-1}\left[2\left(x_{e}+\bar{\varrho} \frac{\mathrm{d}}{\mathrm{d} \bar{\varrho}}\right) \varphi(\bar{\varrho})\right. \\
& \left.-\left(2 x_{e}-\frac{1}{\nu}+\bar{\varrho} \frac{\mathrm{d}}{\mathrm{d} \bar{\varrho}}\right) \mu(\bar{\varrho})\right]
\end{aligned}
$$

For later reference we note results for ideal chains which are also contained in the above expressions. For ideal chains the $n$ components of the $n$-vector model decouple, and Eqs. (A1), (A2), and (A5) can be used within a one-component field theory with Hamiltonian $H=\int \mathrm{d} \mathbf{r}^{\prime \prime}\left[(\nabla \Phi)^{2}+t \Phi^{2}\right] / 2$ and Dirichlet $^{4}$ boundary condition $\Phi=0$ on the surface of the embedded particle. Here $t$ is the Laplace conjugate to the "chain length" $\mathcal{R}_{x}^{2} / 2, \Psi=\Phi^{2}$, and $I, N$, and $N^{\prime}$ are the $n$-independent amplitudes of the $n$-component theory in the absence of anharmonicities. For ideal chains the scaling dimensions of $T_{\|\|}$and $\partial_{\|}^{2} \Phi^{2}$ are degenerate and equal to $d$. It is convenient to introduce ${ }^{8}$ operators $O_{\mathrm{VI}}=\left(\partial_{\|} \Phi\right)^{2} / 2$ and $O_{\mathrm{VII}}$ $=\Phi\left(\partial_{\|}^{2} \Phi\right) / 2$ so that $N T_{\|\|}+N^{\prime} \partial_{\|}^{2} \Phi^{2}=\beta_{\mathrm{VI}} O_{\mathrm{VI}}+\beta_{\mathrm{VII}} O_{\mathrm{VII}}+$ iso, with "iso" denoting isotropic operators, and

$$
\beta_{\mathrm{VI}}=4 N^{\prime}+N d /(d-1), \quad \beta_{\mathrm{VII}}=4 N^{\prime}-N(d-2) /(d-1) .
$$

The reason is that $O_{\mathrm{VI}}$ does not contribute to $\mathcal{E}$ and $\mathcal{M}$, and

$$
\begin{aligned}
& \left\{\mathcal{E}_{\text {aniso }}+\mathcal{E}_{\text {aniso }}^{\prime}, \mathcal{M}_{\text {aniso }}+\mathcal{M}_{\text {aniso }}^{\prime}\right\} \\
& \quad=-\{1,2\} \beta_{\mathrm{VII}} d(d-2)\left(\widetilde{S}_{d} / 2\right) \frac{1}{r^{d}} \frac{r_{\|}^{2}}{r^{2}},
\end{aligned}
$$

with $\widetilde{S}_{d}=\Gamma((d / 2)-1) /\left(4 \pi^{d / 2}\right)$. This is consistent with Eqs. (2.9) and (2.10), since $x_{m}=d-2, x_{e}=x_{m} / 2$, and

$$
\left\{c_{e}, c_{m}\right\}=2 \widetilde{S}_{d}\{1,2\}
$$

follows from Eq. (A4) in the present Gaussian model. We also note that in $d=3$,

$$
\begin{aligned}
& \eta(\varrho)=\frac{1}{(2 \pi)^{3 / 2}} e^{-\varrho^{2} / 2}, \quad \varphi(\varrho)=\frac{1}{2 \pi \varrho} \operatorname{erfc}\left(\frac{\varrho}{\sqrt{2}}\right), \\
& \mu(\varrho)=\frac{4}{\pi \varrho} \mathrm{i}^{2} \operatorname{erfc}\left(\frac{\varrho}{\sqrt{2}}\right) .
\end{aligned}
$$

Due to Eq. (A16) the height contours for $\sigma \ll r \ll \mathcal{R}_{x}$ have the same prolate/oblate character as the particle if the chains are ideal. The reason is that $\beta_{\mathrm{VII}}$ has the sign of $N^{\prime}$, i.e., is positive/negative for prolate/oblate particles. For ellipsoids and dumbbell/lenses this follows from the results of the first and second papers, respectively, in Ref. 8. For an oblate ellipsoid see also Eq. (3.11).

The amplitudes $c_{e}$ and $c_{m}$ also determine the leading isotropic variation for $\sigma \ll r \ll \mathcal{R}_{x}$ [see Eq. (2.8)]. For example, consider $\mathcal{M}$ for self-repelling chains in $d=2$, where

$$
\mathcal{M}_{\text {iso }}-1=-\frac{1}{r^{x_{m}}} \mathcal{I} 2^{-x_{m}}\left|B_{2}\right|,
$$

with

$$
2^{-x_{m}}\left|B_{2}\right|=\left(-\frac{C_{\epsilon \epsilon \epsilon}}{B_{\epsilon}^{3 / 2}} \mathcal{A}_{\epsilon}\right)_{n=0} .
$$

Here we have used the expression for $c_{m}$ in Eq. (A4), the expression for $B_{2}$ in Eq. (A24) of Ref. 3, and have related $I$ and $\Psi$, respectively, to the coefficient $\mathcal{I}$ and the energy density $\epsilon$ with half space amplitude $\mathcal{A}_{\epsilon}$ as given in Ref. 3. The universal number $\left|B_{2}\right|$ is the one in Eq. (3.9). Substituting in Eq. (A19) the explicit forms of $\mathcal{I}$ for an ellipse and a dumbbell or lens, as given in Eqs. (3.5) and (3.8) of Ref. 3, yields expressions consistent with our profiles [Eqs. (3.3) and (3.12)]. 


\section{APPENDIX B: LARGE DISTANCE BEHAVIOR OF LONG CHAIN LIMIT}

Here we show that the expressions on the right hand sides of Eqs. (2.8)-(2.10) not only follow from the small particle expressions (2.5)-(2.7) in the limit $r \ll \mathcal{R}_{x}$ but also from the long chain expressions (3.1) and (3.2), in the limit $\sigma \ll r$. In the latter case we insert the operator expansion (A5) into the long chain expressions. This leads to three-point functions in the bulk and at the critical point which can be taken, e.g., from Appendix A of Ref. 14.

This implies, for example, that the leading isotropic behavior of the monomer density [Eq. (3.2)] determined by the $I$ term is given by $-I r^{-x_{m}} C_{\Psi \Psi \Psi} / B_{\Psi}$ and thus by the second expression in Eq. (2.8), since the three-and two-point amplitudes $C_{\Psi \Psi \Psi}$ and $B_{\Psi}$ also determine the short distance amplitude $c_{m}$, [see Eq. (A4)].

The two anisotropic contributions [Eqs. (2.9) and (2.10)] follow in a similar way. In the case of the $N$ contribution one uses that

$$
-\frac{C_{\mathcal{O O T}}}{B_{\mathcal{O}}}=\frac{d}{d-1} \frac{1}{\Omega_{d}} x_{\mathcal{O}}
$$

where $\mathcal{O}$ equals $\Phi$ or $\Psi, x_{\Phi} \equiv x_{e}, x_{\Psi} \equiv x_{m}$, and $1 / \Omega_{d}$ $=\Gamma(d / 2) /\left(2 \pi^{d / 2}\right)$. Equation (B1) follows, e.g., from the "shift identity",

$$
\begin{gathered}
\int \mathrm{d} \mathbf{x}\left\langle T_{z z}(\mathbf{x}, 0) \mathcal{O}\left(0, z_{1}\right) \mathcal{O}\left(0, z_{2}\right)\right\rangle_{\text {bulk }} \\
=\partial_{z_{1}}\left\langle\mathcal{O}\left(0, z_{1}\right) \mathcal{O}\left(0, z_{2}\right)\right\rangle_{\text {bulk }},
\end{gathered}
$$

on inserting the form ${ }^{14}$ of the bulk correlations at the critical point. Here $z_{1}>0, z_{2}<0$, and $\mathbf{x}$ is the $d-1$ dimensional com- ponent of $\mathbf{r}=(\mathbf{x}, z)$ perpendicular to the $z$ axis.

${ }^{1}$ Soft Matter, edited by G. Gompper and M. Schick (Wiley-VCH, Weinheim, 2005).

${ }^{2}$ R. Tuinier, in Physics meets Biology: from Soft Matter to Cell Biology, edited by G. Gompper, U. B. Kaupp, J. K. Dhont, D. Richter, and R. Winkler (Forchungszertrum Jülich, Jülich, 2004), Chap. C4.

${ }^{3}$ E. Eisenriegler, J. Chem. Phys. 124, 144912 (2006).

${ }^{4}$ P. G. de Gennes, Scaling Concepts in Polymer Physics (Cornell University Press, Ithaca, NY, 1979).

${ }^{5}$ P. M. Johnson, C. M. van Kats, and A. van Blaaderen, Langmuir 21, 11510 (2005).

${ }^{6}$ Our polymers are flexible, with a persistence-length that is much smaller than $\sigma$ and $\mathcal{R}_{x}$. The nanoparticle case or "protein limit") with $\sigma \ll \mathcal{R}_{x}$ has been investigated theoretically to a lesser extent than the "colloid limit" with $\sigma \gg \mathcal{R}_{x}$. This refers to both analytical and simulation approaches.

${ }^{7}$ In terms of the amplitudes $\mathcal{I}, \mathcal{N}$, and $\mathcal{N}^{\prime}$, and $A$ in Ref. 3, our amplitudes $I, N$, and $N^{\prime}$ in Eq. (2.1) $\operatorname{read} I=\mathcal{I} 2^{-x_{m}} A, N=\mathcal{N}$, and $N^{\prime}=\mathcal{N}^{\prime} 2^{-x_{m}} A$, so that $\mathcal{I} \psi=I \Psi, \mathcal{N} T_{k l}=N T_{k l}$, and $\mathcal{N}^{\prime} \partial_{k} \partial_{l} \psi=N^{\prime} \partial_{k} \partial_{l} \Psi$ in the Boltzmann factor of the small particle [see Eqs. (2.2), (2.3), and (2.8) in Ref. 3]. For selfavoiding chains in $d=2$, besides $N^{\prime}$ in Eq. (2.1), there is another next to leading amplitude of anisotropy $\mathcal{N}^{\prime \prime}$ with the same scaling dimension [see Eqs. (3.1) and (3.2) in Ref. 3.]

${ }^{8}$ E. Eisenriegler, A. Bringer, and R. Maaßen, J. Chem. Phys. 118, 8093 (2003); E. Eisenriegler and A. Bringer, J. Phys.: Condens. Matter 17, S1711 (2005)

${ }^{9}$ The competition between $N$ and $N^{\prime}$ in the polymer density profiles around a single anisotropic particle is not found in the polymer-induced orientational interaction with a close wall, where $N$ and $N^{\prime}$ act in the same direction (Ref. 3).

${ }^{10}$ A. Hanke, E. Eisenriegler, and S. Dietrich, Phys. Rev. E 59, 6853 (1999); E. Eisenriegler, J. Phys.: Condens. Matter 12, A227 (2000).

${ }^{11}$ In accordance with Eq. (3.2), the quantities $q=q(\mathbf{r})$ and $\xi=\xi(\mathbf{r})$ in Secs. III A and III B follow from $q=q\left(\mathbf{r}_{1}, \mathbf{r}_{2}\right)$ and $\xi=\xi\left(\mathbf{r}_{1}, \mathbf{r}_{2}\right)$ in Appendix A2 of Ref. 3 on replacing $\mathbf{r}_{1}$ by $\mathbf{r}$ and taking the limit $\mathbf{r}_{2} \rightarrow \infty$.

${ }^{12}$ T. W. Burkhardt, E. Eisenriegler, and I. Guim, Nucl. Phys. B 316, 559 (1989).

${ }^{13}$ E. Eisenriegler, J. Chem. Phys. 113, 5091 (2000).

${ }^{14}$ E. Eisenriegler, J. Chem. Phys. 121, 3299 (2004). 